\title{
Alteration of Theta Timescale Dynamics of Hippocampal Place Cells by a Cannabinoid Is Associated with Memory Impairment
}

\author{
David Robbe ${ }^{1,2}$ and György Buzsáki ${ }^{1}$ \\ ${ }^{1}$ Center for Molecular and Behavioral Neuroscience, Rutgers, The State University of New Jersey, Newark, New Jersey 07102, and 2Institut d'Investigacions \\ Biomediques August Pi i Sunyer, 08036 Barcelona, Spain
}

\begin{abstract}
The integrity of the hippocampus is critical for both spatial navigation and episodic memory, but how its neuronal firing patterns underlie those functions is not well understood. In particular, the modality by which hippocampal place cells contribute to spatial memory is debated. We found that administration of the cannabinoid receptor agonist CP55940 (2-[(1S,2R,5S)-5-hydroxy-2-(3-hydroxypropyl)cyclohexyl]-5-(2-methyloctan-2-yl)phenol) induced a profound and reversible behavioral deficit in the hippocampus-dependent delayed spatial alternation task. On the one hand, despite severe memory impairment, the location-dependent firing of CA1 hippocampal place cells remained mostly intact. On the other hand, both spike-timing coordination between place cells at the theta timescale and theta phase precession of spikes were reversibly reduced. These results raise the possibility that cannabinoids impair memory primarily by altering short-term temporal dynamics of hippocampal neurons. We hypothesize that precise temporal coordination of hippocampal neurons is necessary for guiding behavior in spatial memory tasks.
\end{abstract}

\section{Introduction}

The hippocampus supports both spatial navigation and episodic memory, but how these functions relate to the firing patterns of hippocampal neurons is not well understood (Eichenbaum et al., 1999; Buzsáki, 2005; O’Keefe and Burgess, 2005; Mizumori, 2006). On the one hand, overwhelming evidence supports the early observation (O'Keefe and Dostrovsky, 1971) that hippocampal pyramidal cells fire in particular locations of the environment (Muller, 1996) and movement trajectories can be predicted from the population activity of these "place cells" (Wilson and McNaughton, 1993). Such unit recording studies, combined with lesion experiments, are consistent with the cognitive map theory in which the collective activity of hippocampal place cells reflects an internal representation of the environment, which can be used by the rest of the brain to assist spatial navigation (O'Keefe and Nadel, 1978). On the other hand, recent studies have challenged the view that hippocampal neurons carry only spatial information by reporting that firing patterns of hippocampal neurons can be modulated by nonspatial events (Markus et al., 1995; Hampson et al., 1999; Wood et al., 1999, 2000; Frank et al., 2000; Ferbinteanu and

\footnotetext{
Received May 22, 2009; revised July 14, 2009; accepted Aug. 24, 2009.

This work was supported by National Institutes of Health Grants NS034994 and MH54671, National Science Foundation Grant SBE 0542013, the J. D. MCDonnell Foundation (G.B.), the Human Frontier Science Program, and the Ramón y Cajal Young Investigator Program (D.R.). We thank A. Amarasingham, K. Diba, C. Geisler, K. Mizuseki, E. Pastalkova, and A. Sirota for valuable discussions, comments on this manuscript, and support with data analysis.

Correspondence should be addressed to either of the following: David Robbe, Institut d'Investigacions Biomediques August Pi i Sunyer, Carrer Corsega, 176,08036 Barcelona, Spain, E-mail: davrobbe@clinic.ub.es; or György Buzsáki, Center for Molecular and Behavioral Neuroscience, Rutgers University, 197 University Avenue, Newark, NJ 07102, E-mail: buzsaki@axon.rutgers.edu.

DOI:10.1523/JNEUROSCI.2407-09.2009

Copyright $\odot 2009$ Society for Neuroscience $\quad$ 0270-6474/09/2912597-09\$15.00/0
}

Shapiro, 2003; Pastalkova et al., 2008). These findings have been proposed to provide coding mechanisms underlying the hippocampal contribution to episodic memory (Eichenbaum, 2004; Squire et al., 2004; Hasselmo, 2005; Mizumori, 2006; Shapiro et al., 2006). In addition to discharge rate-based coding, the hippocampus exhibits a clear example of temporal coding characterized in the reliable relationship between the position of the rat and the phase of place cell spikes relative to hippocampal theta oscillations (O'Keefe and Recce, 1993). From the apparent independence of rate and phases of place cells spikes (Hirase et al., 1999; Huxter et al., 2003), it has been proposed that the navigation and memory functions of the hippocampus were supported by theta spike-timing and firing rate mechanisms, respectively (O'Keefe et al., 2005).

The postulated existence of different coding mechanisms underlying hippocampal functions raises the question of their respective behavioral relevance. To examine the relationship between memory, spatial representation, and firing patterns of hippocampal neurons, we analyzed place cell activity recorded from the CA1 region in rats engaged in a hippocampusdependent spatial memory task (delayed alternation in a modified T-maze), and compared them to firing patterns obtained during a behavioral impairment induced by systemic injection of the synthetic cannabinoid 2-[(1S,2 R,5S)-5-hydroxy-2-(3-hydroxypropyl)cyclohexyl]-5-(2-methyloctan-2-yl)phenol (CP55940). This pharmacological tool was chosen because cannabinoids impair hippocampus-dependent memories in humans (Miller and Branconnier, 1983; Curran et al., 2002; Ilan et al., 2004) and rats (Lichtman et al., 1995; Lichtman and Martin, 1996; Hampson and Deadwyler, 1998, 2000) and interfere with the synchrony of hippocampal network activity (Constoe et al., 1975; Hájos et al., 
2000; Robbe et al., 2006, Hajós et al., 2008). We found that, although CP55940 impaired the rat's performance in the spatial memory task, the location-dependent firing of hippocampal neurons was mostly preserved. In contrast, temporal aspects of firing patterns at the theta timescale were altered under the influence of the drug, proportionally with the magnitude of the behavioral alteration. These results raise the possibility that impairment of cell assembly coordination underlies the observed memory deficit.

\section{Materials and Methods}

All protocols were approved by the Institutional Animal Care and Use Committee of Rutgers University. Full methods are available online as supplemental detailed Methods (available at www.jneurosci.org as supplemental material).

Behavioral task. Five Long-Evans rats (male; 300-400 g) were water deprived and trained to perform a hippocampus-dependent delayed T-maze alternation task (see Fig. 1) (Ainge et al., 2007; Pastalkova et al., $2008)$. Once animals performed the task very well $(>85 \%)$ for several consecutive sessions, access to water was provided for a full day before surgery.

Experimental protocol and cannabinoid injection. After recovery from surgical implantation of tetrode array or silicon probe, reestablishment of accurate/stable performance of the alternation task and recording of CA1 ripple local field potential (LFP) oscillations and large amplitude units in the home cage, recordings were performed in the maze before and after a systemic injection of a synthetic cannabinoid [CP55940; potent cannabinoid receptor agonist; $0.1 \mathrm{mg} / \mathrm{kg}$ body weight in a vehicle composed of 5\% ethanol, 5\% Cremophor (a derivative of castor oil and ethylene oxide; Sigma-Aldrich), and 90\% saline]. Vehicle injection had no effect either on behavior performance or on neuronal activity (supplemental Fig. 10, available at www.jneurosci.org as supplemental material).

Place field quantification. To compute firing maps, the space visited by the animal in the maze was divided into a grid of $150 \times 150$ pixels $(1$ pixel $=1 \mathrm{~cm}^{2}$ ), and in each pixel the number of spikes fired was divided by the amount of time an animal spent in that pixel. Multiple successive sessions recorded in a given condition (control, drug, or recovery) were concatenated to generate a single firing map per condition. The resulting firing maps were smooth with a Gaussian kernel of horizontal and vertical SD of 2.4 pixels. Place fields were automatically detected (see supplemental detailed Methods, available at www.jneurosci.org as supplemental material).

Spatial information content. The spatial information content was calculated according to the following equation:

$$
\text { Information per spike }=\sum_{i} P_{i} \cdot \frac{R_{i}}{R} \cdot \log _{2} \frac{R_{i}}{R},
$$

where $i$ is the pixel number, $P_{i}$ is the probability of occupancy of pixel $i, R_{i}$ is the mean firing rate in pixel $i$, and $R$ is the overall mean firing rate (Skaggs et al., 1993).

Spatial correlation. For each place cell, the pixel-by-pixel correlation coefficient between control and drug firing rate maps was calculated. Only pixels in which the rats spent at least $10 \mathrm{~ms}$ in both control and drug sessions were used to generate the correlation coefficients. This correlation coefficient was compared with the pixel-by-pixel correlation between firing maps generated from the first and second halves of the control recording. A similar procedure was performed between recovery and drug firing maps.

Phase precession. Instantaneous theta phase was derived from the Hilbert transform of the filtered $(4-12 \mathrm{~Hz})$ LFP. To estimate the strength of phase precession of place fields, the phases of the spikes were systematically rotated by steps of $1^{\circ}$. For each of the 360 rotations, the correlation between spikes phase and position was quantified using a Spearman rank correlation. The correlation between phase and position for the rotation that gave the regression line with the most negative coefficient was taken as indicator of phase precession.
Theta timescale correlation. To calculate the theta timescale lag between the spikes of two overlapping place fields, cross-correlograms were computed and filtered $(1-20 \mathrm{~Hz})$. The theta timescale lag was determined as the time of the cross-correlograms peak with highest amplitude between -100 and $100 \mathrm{~ms}$.

\section{Results}

\section{Cannabinoid reversibly impairs choice accuracy and} decreases running speed

LFP and multiple single units (supplemental table and Fig. 1, available at www.jneurosci.org as supplemental material) were recorded from the CA1 pyramidal layer of five rats trained to perform a hippocampus-dependent, delayed spatial alternation task (supplemental detailed Methods, available at www.jneurosci. org as supplemental material) (Ainge et al., 2007; Pastalkova et al., 2008). In this task, a rat must remember its previous choice to turn into the arm opposite to the one he visited in the preceding trial (Fig. 1A). Return to the previously visited arm was not rewarded and was regarded as error. After an error trial, rats kept alternating from the erroneous choice. Behavioral/recording sessions lasted for 15-20 min. Experiments began by recording one to three control sessions. After the last control session, rats were injected with $\mathrm{CB}_{1}$ agonist CP55940 (0.1 mg/kg body weight, i.p.), a dose that affected firing patterns and performance of the animal but did not interfere with the ability of the animal to run in the maze (Robbe et al., 2006). Starting one-half of an hour after the injection, one to seven sessions were recorded while the animals were under the effect of the drug. Additionally, one or two recovery sessions were recorded starting $6 \mathrm{~h}$ after the injection or on the following day (supplemental table, available at www.jneurosci. org as supplemental material). In the control condition, rats made very few errors, performing on average at $88 \%$ correct (Fig. $1 B, C$, left panel). After cannabinoid injection, choice errors increased significantly, and behavioral performance was, on average, close to chance level (53\%) (Fig. $1 B, C$, left panel) $(p=$ 0.0001 , two-sided signed rank test). As expected from the well described locomotor effects of cannabinoids (Monory et al., 2007), reduced choice accuracy under the influence of the drug was associated with decreased running speed (Fig. 1D) and fewer trials performed per session (Fig. $1 B, C$, right panel) $(p=$ 0.0002 ). The effect of the cannabinoid injection was reversible both in terms of choice accuracy and running speed (Fig. $1 B-D$ ) ( $p=0.9$ for accuracy and $p=0.8$ for the number of trials per 5 min; control vs recovery). Together, these behavioral observations confirm that, under the influence of cannabinoids, rats fail to perform a spatial memory task. Additionally, this cognitive impairment is associated with slower running speed that will have to be taken into account when analyzing firing patterns of hippocampal neurons.

\section{Spatial modulation of pyramidal cells firing is moderately affected by cannabinoid}

In the hippocampus, the location dependency of pyramidal cell firing is an outstanding and robust neuronal correlate of behavior. However, how these place cells contribute to the memory function of the hippocampus is debated. Different mechanisms have been proposed, including spatial and rate remapping (Colgin et al., 2008). We therefore started by quantifying and comparing basic features of place fields recorded before and after the cannabinoid injection. Of the 14 experiments performed in five rats, one was discarded from additional analysis because the running pattern of the animal was strongly affected by the drug (supplemental Fig. 2, available at www.jneurosci.org as supplemental 
A

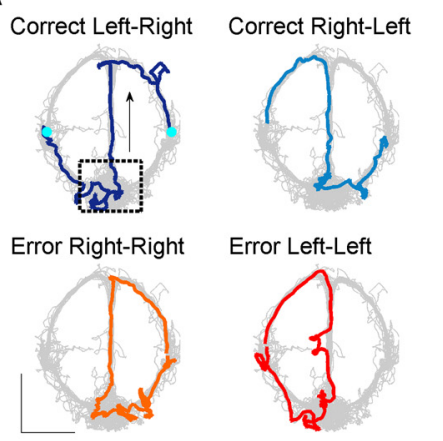

B Control: $92.31 \%$ Correct

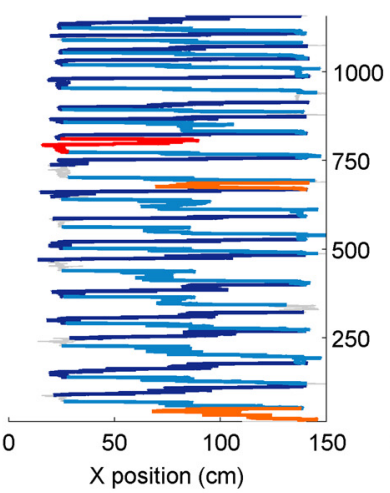

D

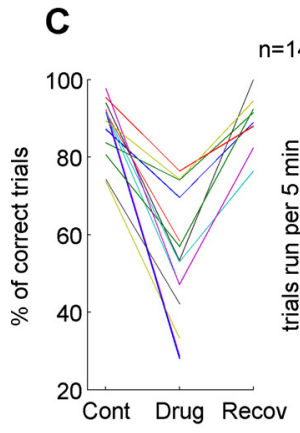

$n=14,5$ rats

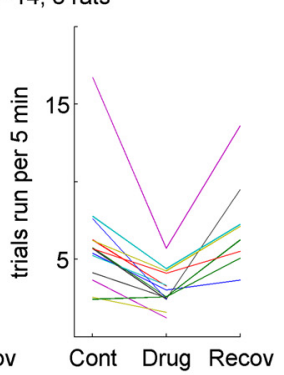

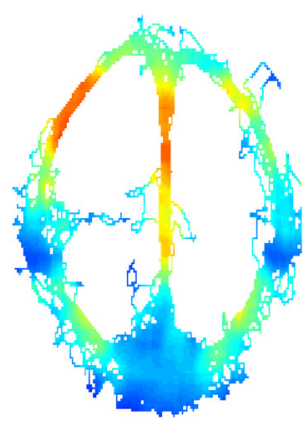

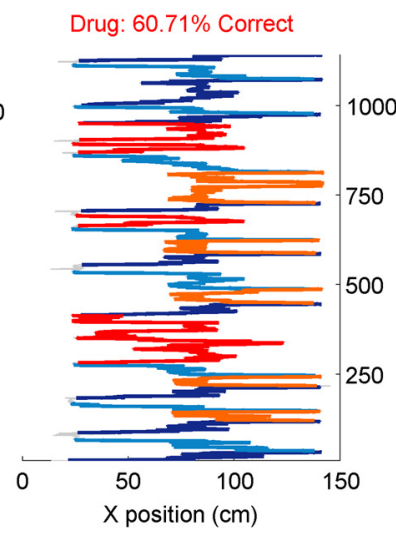

1000

Recovery: $86.84 \%$ Correct
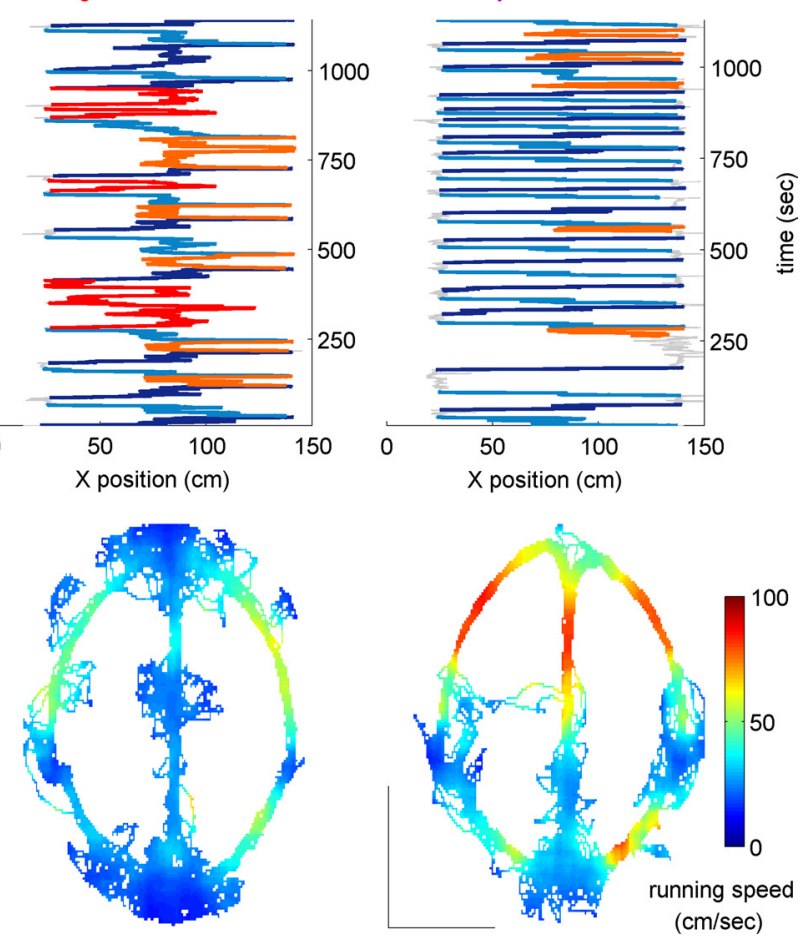

Figure 1. Systemic injection of cannabinoid impaired performance and reduced running speed in a spatial alternation task. $\boldsymbol{A}$, Representative trajectories of a rat performing a delayed spatial alternation task. The top panels illustrate the two possible types of correct trials (left-right, right-left), whereas the bottom panels illustrate the two types of error trials (left-left, right-right). Trials start and finish at the water port (top left panel, blue circles). The delay area is represented by a dashed square. Running direction is indicated by black arrow. The gray lines show trajectories of the rat during the entire session. $\boldsymbol{B}$, Single experiment showing the trajectory of a rat during 20 -min-long sessions recorded one-half of an hour before (control; left), one-half of an hour after (drug; center), and $6 \mathrm{~h}$ (recovery; right) after a systemic injection of the cannabinoid CP55940 (0.1 mg/kg). Correct and error trials are color-coded as in $\boldsymbol{A}$. $\boldsymbol{C}$, Average performance (left; correct trials) and average number of trial per $5 \mathrm{~min}$ (right) for all sessions and rats. $\boldsymbol{D}$, Average color-coded speed maps for the sessions displayed in $\boldsymbol{B}$. The $y$ - and $x$-scale bars in $\boldsymbol{A}$ and $\boldsymbol{D}$ represent $50 \mathrm{~cm}$.

material). In the remaining 13 experiments, 58 putative pyramidal cells were unambiguously recorded through at least two consecutive behavioral conditions (for the number of cells and place fields analyzed per experiment, see supplemental table, available at www.jneurosci.org as supplemental material; for the criteria used to detect place fields, see supplemental detailed Methods, available at www.jneurosci.org as supplemental material). Despite strong impairment in behavioral accuracy, spatial remapping of place cells was not observed in drug condition, as quantified by the spatial correlation measure of place cells (see Materials and Methods) (Fig. $2 A, B)(p=0.23$, two-sided signed rank test) [supplemental Fig. 4 (available at www.jneurosci.org as supplemental material) shows the firing maps of all the place cells reliably tracked across experimental conditions]. The spatial information content of the firing of the place cells was not significantly affected either (Fig. 2C) $(p=0.38)$. Similarity of position coding by the place cell was further illustrated by the similar population vectors (Gothard et al., 1996) across control, drug, and recovery conditions (supplemental Fig. 5, available at www.jneurosci.org as supplemental material). We next compared other features of place fields. We observed a small but significant decrease of place field area in drug condition (8\%) (Fig. $2 D)(p<0.0001)$ and a small decrease in the ratio between spikes fired in the field(s) and the total number of spikes fired by the cell (Fig. $2 E)(p=0.01)$. More noticeably, we found a strong decrease of both in-field peak firing rates and in-field average firing rates (Fig. $2 F, G)(p<0.0001)$. Because locomotion speed is known to affect firing rates of pyramidal neurons (McNaughton et al., 1983; Czurkó et al., 1999; Huxter et al., 2003) and the drug effectively reduced the in-field running speed of the rat (Fig. $2 H)(p<0.0001)$, we attempted to quantify the contribution of speed decrease on the observed changes in firing rate. We performed an analysis of covariance between in-field average running speed and both in-field peak and average firing rates. These comparisons revealed that the cannabinoid effect on both peak and average firing rates was essentially explained by the decrease in running speed (Fig. $2 I, J)(p=0.14$ and $p=0.12$, covariance).

Units that could be reliably tracked across conditions represented only one-third of the total number of well isolated cells (supplemental table, available at www.jneurosci.org as supplemental material), whereas the majority of the recorded cells were sufficiently stable within but not across sessions. We used the entire dataset to perform independent population analysis of the place cell features examined above. Place cells analyzed independently in control, drug, and recovery conditions confirmed the observations made on the subset of cells monitored throughout conditions (supplemental Fig. 6, available at www. jneurosci.org as supplemental material). We also noticed that, after cannabinoid injection, the number of putative pyramidal cells clustered per electrodes significantly increased $(p=$ 0.017) (supplemental Fig. 3, available at www.jneurosci.org as supplemental material), suggesting that a fraction of previously silent cells began to emit spikes (Thompson and Best, 1989). Because this effect did not reverse ( $p=0.8$ ) (supplemental Fig. 3, available at www.jneurosci.org as supplemental material) and was not accompanied by an increase in the number of place cells ( $p=0.25)$ (supplemental Fig. 3, available at www.jneurosci.org as supplemental material), it is difficult to relate it to the observed behavioral impairment. This result is reminiscent of the obser- 
vation in head-restrained rats, in which the number of coactive neurons increased after cannabinoid injection and is in support of the hypothesis that neurons tend to "escape" population control after cannabinoid injections (Robbe et al., 2006).

\section{Theta phase precession and theta timescale temporal correlations are altered by cannabinoid}

The previous set of analyses revealed that, despite the strong memory impairment caused by the cannabinoid, place cells firing pattern dynamics at behavioral timescale were mostly preserved, especially if the effect of running speed on firing rate was taken into account. In behaving rodents, the firing rate of hippocampal neurons is strongly modulated by the $5-10 \mathrm{~Hz}$ theta rhythm (Buzsáki, 2002), and thetabased mechanisms have been proposed to support episodic memory (Hasselmo, 2005; Jensen and Lisman, 2005). In headrestrained animals, cannabinoids altered the firing pattern and coordination of hippocampal neurons at short $( \pm 150$ ms) timescale (Robbe et al., 2006). Therefore, we next investigated whether theta timescale dynamics of hippocampal place cells were affected by the cannabinoid administration in the present behavioral setting.

First, autocorrelograms and power spectrograms were computed from the spike trains and LFP recorded inside each place field. Both methods showed a strong drug-induced decrease in the power and frequency of unit oscillation (Fig. $3 A-D$ ) (unit power, $p=0.0002$, two-sided signed rank test; unit frequency, $p<0.0001$ ) and LFP (supplemental Fig. $7 A, B$, available at www.jneurosci.org as supplemental material) in the theta band. In principle, both these changes could be attributed to reduced running speed (Geisler et al., 2007; Jeewajee et al., 2008; Montgomery et al., 2009). An analysis of covariance showed that most of the drug-induced decrease of unit theta power was explained by speed (Fig. $3 E)(p=0.23)$. In contrast, the drug-induced decrease in oscillatory frequency of unit activity remained strongly significant even once the running speed effect was taken into account (Fig. $3 F$ ) $(p<0.0001)$. A similar, speed-independent decrease in theta LFP frequency was also observed (supplemental Fig. 7C, available at www.jneurosci.org as supplemental material). An additional regression analysis between unit and LFP theta frequency revealed that the drug had a distinct effect on those two variables (supplemental Fig. 7D, available at www.jneurosci.org as supplemental material) (the slopes of the linear regression in control and drug in subplots $\boldsymbol{B}-\boldsymbol{H}$ by circles.
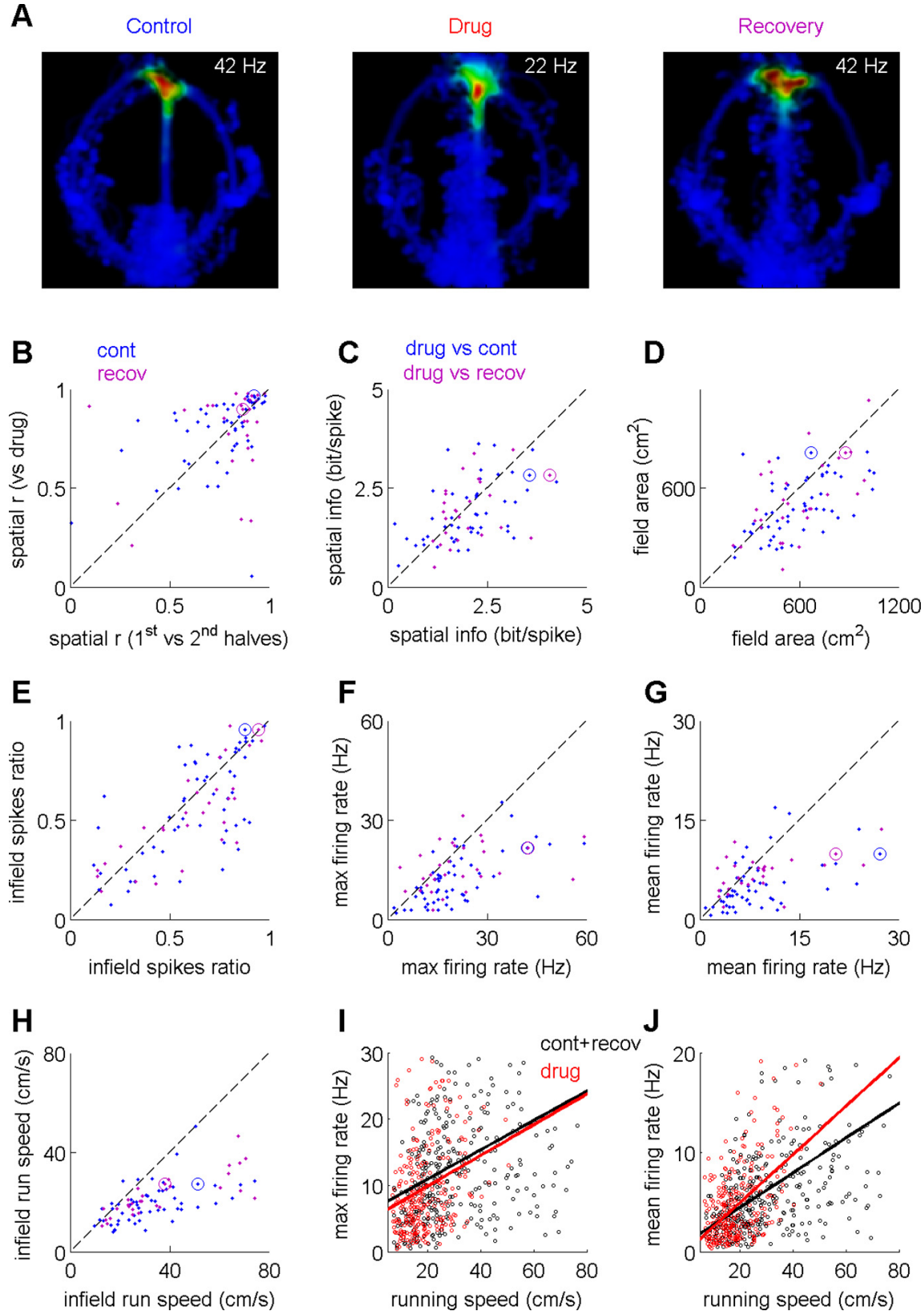

Figure 2. Cannabinoid injection only marginally affects spatial tuning of place cells, and the changes in firing rate can be accounted for by the decrease of running speed. $\boldsymbol{A}$, Representative firing maps of a place cell recorded successively before (left column), 20 min after (central column), and $6 \mathrm{~h}$ (right column) after (P55940 systemic injection. The red color represents peak firing rate (indicated in the top right corner of each firing map), light blue shows low firing rate, and dark blue visited locations. Supplemental Figure 4 (available at www.jneurosci.org as supplemental material) shows the firing maps of all place cells reliably tracked across experimental conditions. $\boldsymbol{B}$, Change in spatial correlation for place cells unambiguously recorded across successive recording sessions. The pixel-by-pixel spatial correlation coefficient was computed between the firing maps of the first and second halves of the control session and compared with the coefficient computed from control and drug firing maps (cont; $n=50$ place cells). A similar comparison was done between drug and recovery recordings (recov; $n=28$ ). $C$, Change in spatial information content (drug vs control are blue dots; drug vs recovery are magenta dots; same $n$ as in $\boldsymbol{B}$ ). Effect of cannabinoid on place field area $(\boldsymbol{D})$, ratio between in-field spikes and all spikes $(\boldsymbol{E})$, in-field peak firing rate $(\boldsymbol{F})$, average firing rate $(\boldsymbol{G})$, and running speed $(\boldsymbol{H})$, for place fields unambiguously recorded across successive conditions (drug vs control, $n=55$ place fields; drug vs recovery, $n=30$ ). Covariance analysis between running speed and peak firing rate $(\boldsymbol{I})$ and running speed and mean firing rate $(\boldsymbol{J})$ for all place fields identified in control plus recovery $(n=368$; black circles) and drug conditions $(n=276)$. The example place cell/fields are marked

conditions were statistically different, $p<0.0001$, analysis of covariance). These results suggest that the cannabinoid, independently of changes in running speed, reduced differentially the oscillatory (theta) frequency of place cells and LFP. 
A

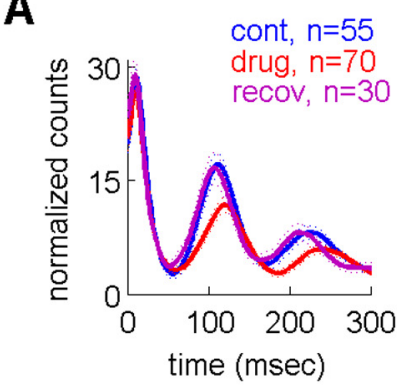

C

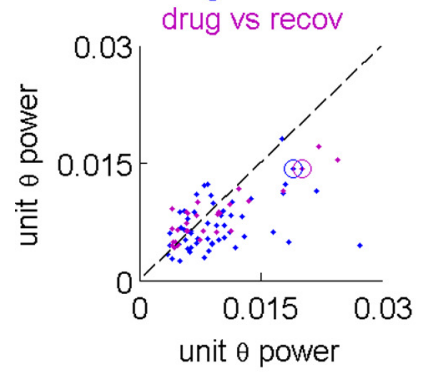

E

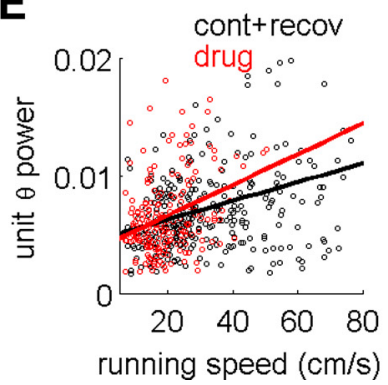

B

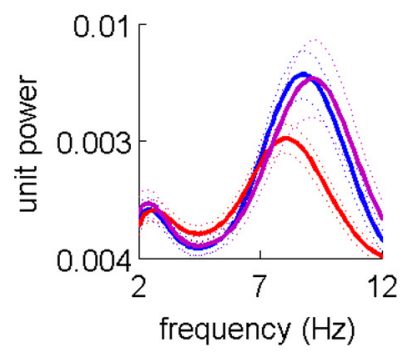

D

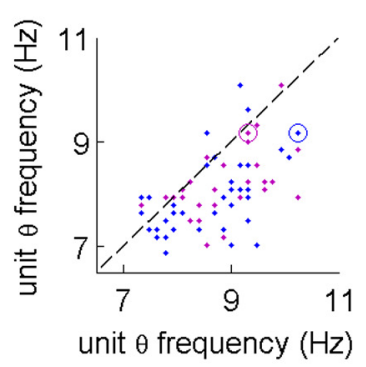

$\mathbf{F}$

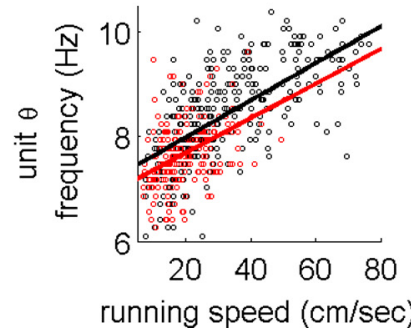

Figure 3. Oscillation frequency of place cells is decreased by cannabinoid. Averaged filtered $(0-20 \mathrm{~Hz})$ autocorrelograms $(\boldsymbol{A})$ and power spectrograms $(\boldsymbol{B})$ from spikes fired in place fields and recorded across at least two conditions. " $n$ " represents different place fields. The dotted lines represent SEM. Change in unit theta power $(\boldsymbol{C})$ and theta frequency $(\boldsymbol{D})$ for place fields tracked across conditions (drug vs control, $n=55$; drug vs recovery, $n=30$ ). Covariance analysis between running speed and peak theta power $(\boldsymbol{E})$ and running speed and peak theta frequency $(\boldsymbol{F})$, for place fields showing a local maxima in their power spectrogram between 5 and $12 \mathrm{~Hz}$ (control plus recovery, $n=316$; drug, $n=200$ ). Example place fields from Figure 1 are marked in subplots $\boldsymbol{C}$ and $\boldsymbol{D}$ by circles.

When rats perform in a maze, the theta oscillation frequency of place cells is faster than that of the ongoing LFP (theta) oscillation, resulting in a gradual shift of their spikes relative to the simultaneously recorded phase of theta (phase precession) (O'Keefe et al., 1993). Therefore, a direct measure of the influence of the drug on the spike-LFP theta temporal relationship can be obtained by comparing the strength of the phase precession (see Materials and Methods), a measurement that has the advantage of being independent of running speed (O'Keefe et al., 1993; Skaggs et al., 1996; Huxter et al., 2003; Geisler et al., 2007; Diba and Buzsáki, 2008). To reduce the possibility that changes in overt behavior of the animal biased the phase precession analysis, spikes fired while animals were rearing and running at a speed $<5 \mathrm{~cm} / \mathrm{s}$ were discarded from the analysis. Both the correlation coefficient and the slope of the linear regression between spikes phase and position of the animal were decreased under the influence of cannabinoid (Fig. 4A-C) (correlation coefficient, $p<0.0001$; slope, $p=$ 0.01 , two-sided signed rank test). To determine whether this result was related to phase estimation errors, caused by de-

creased LFP theta power, we examined, for each place field recorded across experimental conditions, the correlation between the change in phase precession coefficient and the change in theta LFP power but did not find a significant effect (Spearman's rank correlation coefficient, $r=-0.11 ; p=0.38$ ).

To further investigate how theta timescale temporal coding was altered by the cannabinoid, we took advantage of the observation that, within the theta cycle, the time lag between the spikes of overlapping place cells is correlated with the spatial distance between the respective place fields. Thus, this theta-scale time lag provides information about the past, present, and future positions of the animal (Skaggs et al., 1996; Dragoi and Buzsáki, 2006). The distance versus theta-scale time lag correlation is referred to as the sequence compression index and has been shown to be independent of the running speed (Geisler et al., 2007; Diba et al., 2008). We compared theta-scale sequence compression index in control, drug, and recovery conditions in place cell pairs with overlapping spatial representations (see Materials and Methods). This analysis was performed using only the spikes fired inside the overlapping place fields. Similarly to the phase precession analysis, spikes fired while animals reared and ran at a speed $<5 \mathrm{~cm} / \mathrm{s}$ were discarded. The correlation between theta timescale time lag and place fields distance was strongly and reversibly reduced after cannabinoid injection (Fig. 5A,B) (correlation coefficient between field distance and theta time lag was $0.83,0.41$, and 0.83 for control, drug, and recovery, respectively; control vs drug, $Z$ score $=3.06$; drug vs recovery, $Z$ score $=2.42$; and control vs recovery, $Z$ score $=0.50$ permutation test) (permutation test is described in supplemental detailed Methods, available at www.jneurosci.org as supplemental material) (see also supplemental Fig. 9, available at www.jneurosci.org as supplemental material). To understand the origin of this increased temporal jitter in the drug condition, we calculated the power spectrogram of the unit pair cross-correlograms and averaged them to obtain a group measure for each condition. A strong and reversible decrease in both power and frequency of the crosscorrelograms was caused by the drug (Fig. 5C) (control vs drug and recovery vs drug, $Z$ score $>2$ for all frequencies between 8 and $12 \mathrm{~Hz}$, permutation test). In a separate analysis, we constrained our analysis to a subset of cell pairs, whose crosscorrelograms were theta modulated (see supplemental detailed Methods, available at www.jneurosci.org as supplemental material). In this subset of cell pairs, the correlation remained significantly smaller in the drug condition (supplemental Fig. 8, available at www.jneurosci.org as supplemental material) (control vs drug, $Z$ score $=4.96$; recovery vs drug, $Z$ score $=3.52$; control vs recovery, $Z$ score $=0.32$; permutation test). Together, this analysis showed that theta timescale coordination between spikes of neuron pairs is reversibly reduced by cannabinoid administration. Finally, in two experiments (rat 5) (supplemental table, available at www.jneurosci.org as supplemental material), sufficiently large numbers of overlapping pairs of place fields were recorded in many successive sessions. In these datasets, a strong correlation was found between the percentage of correct trials and theta-scale sequence compression index (Fig. 5D) $(r=$ $0.82 ; p<0.0001$ ), further supporting the hypothesis that alteration in theta timescale spike coordination is linked to the cognitive impairment.

\section{Discussion}

We found that cannabinoid administration resulted in a profound behavioral deficit in a hippocampus-dependent task. De- 
spite the severe memory impairment, the location-dependent firing of place cells was mostly spared, whereas both spiketiming coordination between place cells at the theta timescale and theta phase precession of spikes were reversibly reduced. These findings imply that spatial memory depends primarily on the temporal coordination of neurons and can be dissociated from the stability of the spatial map.

\section{Spatial representation is mostly preserved under the influence of cannabinoid}

According to the cognitive map theory (O'Keefe and Nadel, 1978), positiondependent activity of hippocampal neurons represents an internal map, which enables flexible spatial navigation and assists in solving hippocampus-dependent spatial memory tasks (O'Keefe and Speakman, 1987; Lenck-Santini et al., 2001, 2002; Cacucci et al., 2008), such as the delayed spontaneous alteration task, used in the present experiment. From this perspective, an unexpected finding of our experiment is that administration of the cannabinoid receptor agonist CP55940 left the spatial map representation mostly intact, while inducing a severe memory deficit. No spatial "remapping" of place cell activity was observed under the influence of the drug either by visual inspection of the firing maps or by using the quantitative pixel-by-pixel correlation method. The spatial resolution of the place cells did not deteriorate as shown by the stable information content of individual place cells, and the similar population vectors across conditions. In addition to these preserved spatial parameters, we also found a reversible decrease of both average and peak firing rates of place fields after cannabinoid administration. However, it is unlikely that changes in firing rate of place cells were responsible for the memory deterioration (Colgin et al., 2008) because the rate decrease was accounted for by the decreased running speed within the individual place fields (McNaughton et al., 1983; Czurkó et al., 1999; Huxter et al., 2003). In addition, it has been shown previously that reduced firing rates during slower runs in intact rats do not affect the quality of place fields. In summary, the profound drug-induced memory deficit was not correlated with detectable changes in the spatial map and the underlying behavioral-scale temporal dynamic.

Our findings complement a previous experiment, which addressed the relationship between spatial memory and hippocampal map representation. Jeffery and al. (2003) trained rats on a tone-cued spatial navigation task in a black box and tested them later in a white box in the same room. Although the change from black to white induced remapping of most place cells, navigational performance remained mostly above chance level (Jeffery et al., 2003). These findings, combined with ours, are at variance with the widely held view that navigation is guided by the map, maintained by the hippocampal place cells (O'Keefe et al., 1987; Lenck-Santini et al., 2001, 2002; Cacucci et al., 2008). In support of the hippocampal map-dependent navigation, one can argue that an extrahippocampal mechanism maintained memory performance in both the navigation task of Jeffrey et al. and ours. However, this interpretation is not likely since hippocampal lesion essentially abolished performance in both tasks (Jeffery et al., 2003; Ainge et al., 2007; Pastalkova et al., 2008). Furthermore, several observations point to the hippocampus as an important target of systemic cannabinoid injection and the mediator of the cognitive impairment. First, intrahippocampal injection of the same cannabinoid used in our study reproduced the spatial deficits induced by systemic cannabinoid injection (Lichtman et al., 1995). Second, the effects of systemic cannabinoid injection in memory tasks resemble the effects of hippocampal lesions (Hampson and Deadwyler, 1998). Third, recreational (systemic) use of cannabis in humans deteriorates hippocampus-dependent episodic memory (Miller et al., 1983; Curran et al., 2002; Ilan et al., 2004). In conclusion, the mechanisms responsible for the severe memory deficit seen in our experiments are likely to in- 

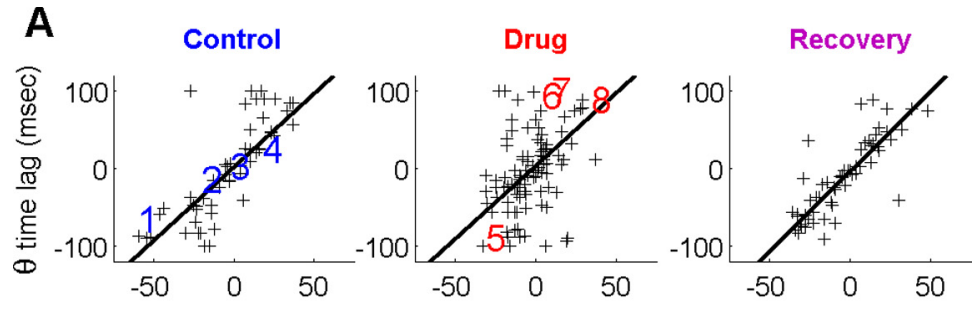

inter-fields distance $(\mathrm{cm})$
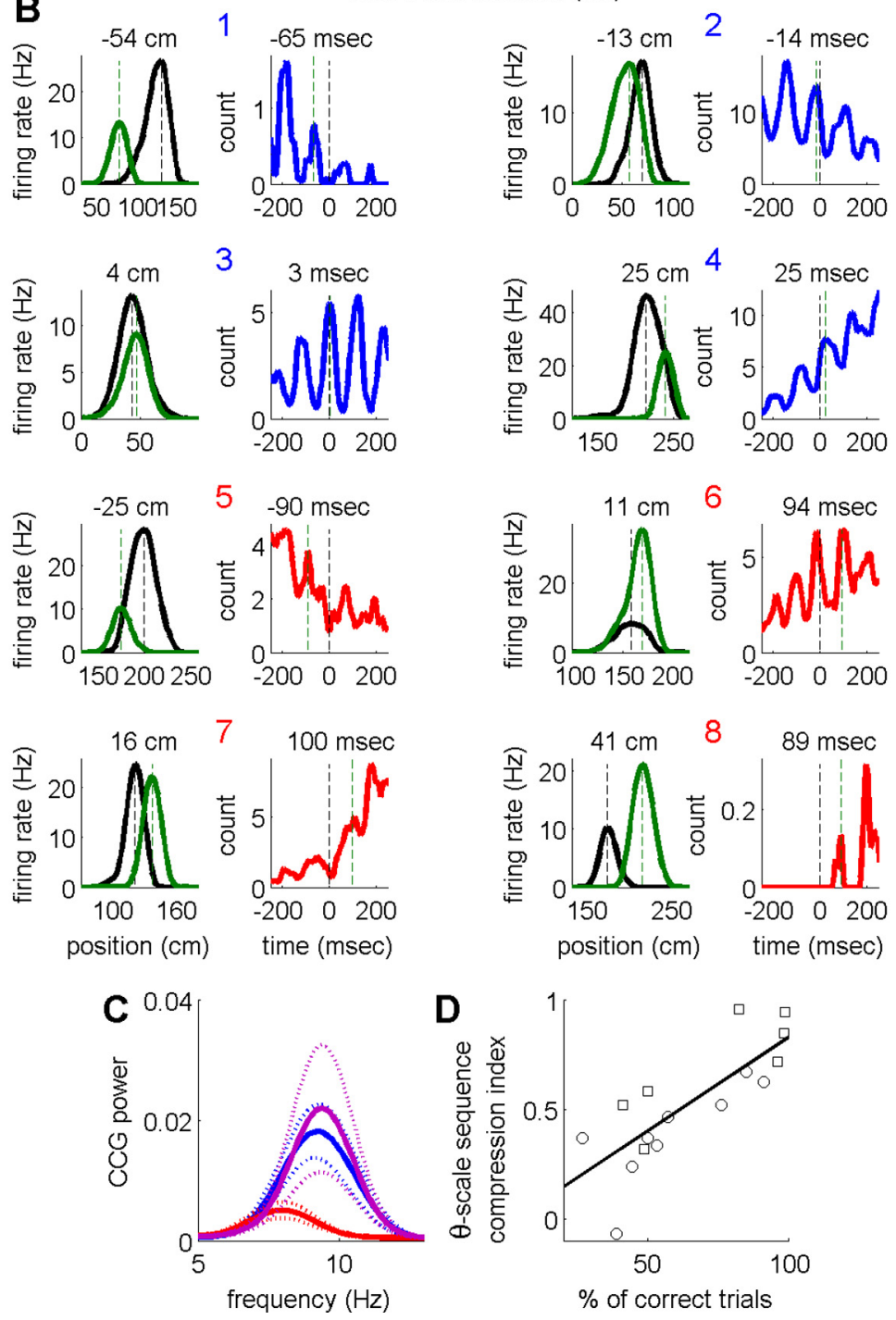

Figure 5. Theta timescale spike coordination is altered by cannabinoid. $A$, Relationship between fields distance and theta-scale time lag for all overlapping neuron pairs recorded in control $(n=56)$, drug $(n=96)$, and recovery conditions $(n=55)$. Numbers refer to neuron pairs shown in $\boldsymbol{B}$. $\boldsymbol{B}$, Examples of neuron pairs in control (1-4) and drug (5-8) conditions (different pairs). The left columns represent the average firing rate along the track, whereas the right columns show the filtered $(0-20 \mathrm{~Hz}) \mathrm{cross}-$ correlograms ( -200 and $200 \mathrm{~ms}$ ). An example pair with overlapping place fields recorded in both control and drug conditions is shown in supplemental Figure 9 (available at www.jneurosci.org as supplemental material). C, Average power of the crosscorrelograms (calculated from \pm 2 s cross-correlograms) for pairs shown in $A$. The dotted lines show SEM. $D$, Correlation between the behavioral performance (percentage of correct trials) and the theta-scale sequence compression index in rat 5 . The different symbols refer to data obtained in two different experiments (experiment 12: circles, $n=9$; experiment 13: squares, $n=7$ ).

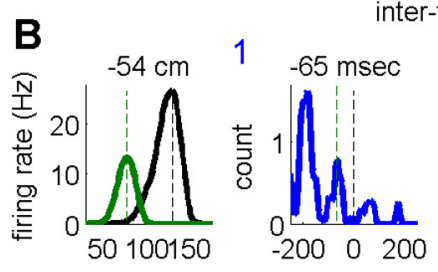

coding mechanisms for hippocampal functions (Skaggs et al., 1996; Hasselmo, 2005; Jensen and Lisman, 2005; O'Keefe et al., 2005). When the rat crosses the place field of a neuron, spikes are fired at progressively earlier phases of the theta LFP oscillations (O'Keefe et al., 1993). Furthermore, place field sequences and distance representation of neurons on the track are represented as temporal (phase) offsets within single theta cycles in a compressed manner (Skaggs et al., 1996; Dragoi et al., 2006). Both of these temporal codes are independent of running speed (O'Keefe et al., 1993; Huxter et al., 2003; Geisler et al., 2007; Diba et al., 2008). Recently, a dual coding mechanism has been proposed, suggesting that the theta phase of spikes would encode spatial position of the rat, whereas the firing rate would be available for encoding episodic memories (Huxter et al., 2003; O'Keefe et al., 2005). At variance with this model, our study shows that representation of position by discharge rates was not affected after cannabinoid injection and memory impairment but both spike phase precession and distance-time compression were altered. In a previous head-restrained study, synchrony of hippocampal neurons at the short timescale $( \pm 150 \mathrm{~ms})$ was severely impaired by cannabinoids, whereas the firing rates of pyramidal cells and interneurons were only mildly affected (Robbe et al., 2006). The current results obtained in behaving rats further support that synchrony is the main network target mechanism of cannabinoids (Constoe et al., 1975; Buonamici et al., 1982; Hájos et al., 2000; Bernard et al., 2005; Robbe et al., 2006; Hajós et al., 2008). Additionally, our findings reinforce the conclusion of a recent study showing that phase precession, theta-scale distance compression and performance in the water maze task were impaired in an animal model of temporal lobe epilepsy (Lenck-Santini and Holmes, 2008). Here, since alteration of short-timescale events coincided with the behavioral impairment, we hypothesize that proper temporal coordination of spikes across cell assemblies is critical for normal spatial memory performance. Theta timescale compression of the representation of past, present, and volve the hippocampus but may be different from those supported by the position-specific firing of place cells.

\section{Cannabinoids affect spike-timing coordination and spatial memory}

In addition to the firing rate-based computation, temporal aspects of place cell activity have been proposed to provide future locations may be needed for bringing these neurons in the temporal window of plasticity (Skaggs et al., 1996; Dragoi et al., 2006). In addition, or alternatively, synchrony may be critical to effectively transfer the content of hippocampal cell assemblies to neocortical targets and/or properly link cell assembly sequences in time (Pastalkova et al., 2008). Yet another possibility is that theta timescale coordination of neuronal firing is necessary to 
convey the hippocampal spatial representation to other structures, which assist in guiding behavioral performance. Although these mechanisms have yet to be elaborated, our findings imply that theta timescale coordination of neuronal activity is critical for memory performance, whereas properly anchored spatial representation is not sufficient.

\section{References}

Ainge JA, van der Meer MA, Langston RF, Wood ER (2007) Exploring the role of context-dependent hippocampal activity in spatial alternation behavior. Hippocampus 17:988-1002.

Bernard C, Milh M, Morozov YM, Ben-Ari Y, Freund TF, Gozlan H (2005) Altering cannabinoid signaling during development disrupts neuronal activity. Proc Natl Acad Sci U S A 102:9388-9393.

Buonamici M, Young GA, Khazan N (1982) Effects of acute delta 9-THC administration on EEG and EEG power spectra in the rat. Neuropharmacology 21:825-829.

Buzsáki G (2002) Theta oscillations in the hippocampus. Neuron 33: 325-340.

Buzsáki G (2005) Theta rhythm of navigation: link between path integration and landmark navigation episodic and semantic memory. Hippocampus 15:827-840.

Cacucci F, Yi M, Wills TJ, Chapman P, O'Keefe J (2008) Place cell firing correlates with memory deficits and amyloid plaque burden in Tg2576 Alzheimer mouse model. Proc Natl Acad Sci U S A 105:7863-7868.

Colgin LL, Moser EI, Moser MB (2008) Understanding memory through hippocampal remapping. Trends Neurosci 31:469-477.

Constoe PF, Jones BC, Chin L (1975) Delta-9-tetrahydrocannabinol, EEG and behavior:the importance of adaptation to the testing milieu. Pharmacol Biochem Behav 3:173-177.

Curran HV, Brignell C, Fletcher S, Middleton P, Henry J (2002) Cognitive and subjective dose-response effects of acute oral Delta 9-tetrahydrocannabinol (THC) in infrequent cannabis users. Psychopharmacology (Berl) 164:61-70

Czurkó A, Hirase H, Csicsvari J, Buzsáki G (1999) Sustained activation of hippocampal pyramidal cells by "space clamping" in a running wheel. Eur J Neurosci 11:344-352.

Diba K, Buzsáki G (2008) Hippocampal network dynamics constrain the time lag between pyramidal cells across modified environments. J Neurosci 28:13448-13456.

Dragoi G, Buzsáki G (2006) Temporal encoding of place sequences by hippocampal cell assemblies. Neuron 50:145-157.

Eichenbaum H (2004) Hippocampus: cognitive processes and neural representations that underlie declarative memory. Neuron 44:109-120.

Eichenbaum H, Dudchenko P, Wood E, Shapiro M, Tanila H (1999) The hippocampus memory and place cells: is it spatial memory or a memory space? Neuron 23:209-226.

Ferbinteanu J, Shapiro ML (2003) Prospective and retrospective memory coding in the hippocampus. Neuron 40:1227-1239.

Frank LM, Brown EN, Wilson M (2000) Trajectory encoding in the hippocampus and entorhinal cortex. Neuron 27:169-178.

Geisler C, Robbe D, Zugaro M, Sirota A, Buzsáki G (2007) Hippocampal place cell assemblies are speed-controlled oscillators. Proc Natl Acad Sci U S A 104:8149-8154.

Gothard KM, Skaggs WE, McNaughton BL (1996) Dynamics of mismatch correction in the hippocampal ensemble code for space: interaction between path integration and environmental cues. J Neurosci 16:80278040.

Hajós M, Hoffmann WE, Kocsis B (2008) Activation of cannabinoid-1 receptors disrupts sensory gating and neuronal oscillation: relevance to schizophrenia. Biol Psychiatry 63:1075-1083.

Hájos N, Katona I, Naiem SS, MacKie K, Ledent C, Mody I, Freund TF (2000) Cannabinoids inhibit hippocampal GABAergic transmission and network oscillations. Eur J Neurosci 12:3239-3249.

Hampson RE, Deadwyler SA (1998) Role of cannabinoid receptors in memory storage. Neurobiol Dis 5:474-482.

Hampson RE, Deadwyler SA (2000) Cannabinoids reveal the necessity of hippocampal neural encoding for short-term memory in rats. J Neurosci 20:8932-8942.

Hampson RE, Simeral JD, Deadwyler SA (1999) Distribution of spatial and nonspatial information in dorsal hippocampus. Nature 402:610 614.

Hasselmo ME (2005) What is the function of hippocampal theta rhythm? Linking behavioral data to phasic properties of field potential and unit recording data. Hippocampus 15:936-949.

Hirase H, Czurkó A, Csicsvari J, Buzsáki G (1999) Firing rate and thetaphase coding by hippocampal pyramidal neurons during "space clamping." Eur J Neurosci 11:4373-4380.

Huxter J, Burgess N, O'Keefe J (2003) Independent rate and temporal coding in hippocampal pyramidal cells. Nature 425:828-832.

Ilan AB, Smith ME, Gevins A (2004) Effects of marijuana on neurophysiological signals of working and episodic memory. Psychopharmacology (Berl) 176:214-222.

Jeewajee A, Lever C, Burton S, O’Keefe J, Burgess N (2008) Environmental novelty is signaled by reduction of the hippocampal theta frequency. Hippocampus 18:340-348.

Jeffery KJ, Gilbert A, Burton S, Strudwick A (2003) Preserved performance in a hippocampal-dependent spatial task despite complete place cell remapping. Hippocampus 13:175-189.

Jensen O, Lisman JE (2005) Hippocampal sequence-encoding driven by a cortical multi-item working memory buffer. Trends Neurosci 28:67-72

Lenck-Santini PP, Holmes GL (2008) Altered phase precession and compression of temporal sequences by place cells in epileptic rats. J Neurosci 28:5053-5062.

Lenck-Santini PP, Save E, Poucet B (2001) Evidence for a relationship between place-cell spatial firing and spatial memory performance. Hippocampus 11:377-390.

Lenck-Santini PP, Muller RU, Save E, Poucet B (2002) Relationships between place cell firing fields and navigational decisions by rats. J Neurosci 22:9035-9047.

Lichtman AH, Martin BR (1996) Delta 9-tetrahydrocannabinol impairs spatial memory through a cannabinoid receptor mechanism. Psychopharmacology (Berl) 126:125-131.

Lichtman AH, Dimen KR, Martin BR (1995) Systemic or intrahippocampal cannabinoid administration impairs spatial memory in rats. Psychopharmacology (Berl) 119:282-290.

Markus EJ, Qin YL, Leonard B, Skaggs WE, McNaughton BL, Barnes CA (1995) Interactions between location and task affect the spatial and directional firing of hippocampal neurons. J Neurosci 15:7079-7094

McNaughton BL, Barnes CA, O'Keefe J (1983) The contributions of position direction and velocity to single unit activity in the hippocampus of freely-moving rats. Exp Brain Res 52:41-49.

Miller LL, Branconnier RJ (1983) Cannabis: effects on memory and the cholinergic limbic system. Psychol Bull 93:441-456.

Mizumori SJ (2006) Hippocampal place fields: a neural code for episodic memory? Hippocampus 16:685-690.

Monory K, Blaudzun H, Massa F, Kaiser N, Lemberger T, Schütz G, Wotjak CT, Lutz B, Marsicano G (2007) Genetic dissection of behavioural and autonomic effects of Delta(9)-tetrahydrocannabinol in mice. PLoS Biol 5:e269.

Montgomery SM, Betancur MI, Buzsáki G (2009) Behavior-dependent coordination of multiple theta dipoles in the hippocampus. J Neurosci 29:1381-1394.

Muller R (1996) A quarter of a century of place cells. Neuron 17:813-822.

O'Keefe J, Burgess N (2005) Dual phase and rate coding in hippocampal place cells: theoretical significance and relationship to entorhinal grid cells. Hippocampus 15:853-866.

O'Keefe J, Dostrovsky J (1971) The hippocampus as a spatial map. Preliminary evidence from unit activity in the freely-moving rat. Brain Res 34:171-175.

O'Keefe J, Nadel L (1978) The hippocampus as a cognitive map. Oxford: Oxford UP.

O'Keefe J, Recce ML (1993) Phase relationship between hippocampal place units and the EEG theta rhythm. Hippocampus 3:317-330.

O'Keefe J, Speakman A (1987) Single unit activity in the rat hippocampus during a spatial memory task. Exp Brain Res 68:1-27. 
Pastalkova E, Itskov V, Amarasingham A, Buzsáki G (2008) Internally generated cell assembly sequences in the rat hippocampus. Science 321:1322-1327.

Robbe D, Montgomery SM, Thome A, Rueda-Orozco PE, McNaughton BL, Buzsáki G (2006) Cannabinoids reveal importance of spike timing coordination in hippocampal function. Nat Neurosci 9:15261533.

Shapiro ML, Kennedy PJ, Ferbinteanu J (2006) Representing episodes in the mammalian brain. Curr Opin Neurobiol 16:701-709.

Skaggs WE, McNaughton BL, Gothard KM, Markus EJ (1993) An information-theoretic approach to deciphering the hippocampal code. Adv Neural Inf Process Syst 5:1030-1037.

Skaggs WE, McNaughton BL, Wilson MA, Barnes CA (1996) Theta phase precession in hippocampal neuronal populations and the compression of temporal sequences. Hippocampus 6:149-172.

Squire LR, Stark CE, Clark RE (2004) The medial temporal lobe. Annu Rev Neurosci 27:279-306.

Thompson LT, Best PJ (1989) Place cells and silent cells in the hippocampus of freely-behaving rats. J Neurosci 9:2382-2390.

Wilson MA, McNaughton BL (1993) Dynamics of the hippocampal ensemble code for space. Science 261:1055-1058.

Wood ER, Dudchenko PA, Eichenbaum H (1999) The global record of memory in hippocampal neuronal activity. Nature 397:613-616.

Wood ER, Dudchenko PA, Robitsek RJ, Eichenbaum H (2000) Hippocampal neurons encode information about different types of memory episodes occurring in the same location. Neuron 27:623-633. 